MFTF-B Refrigerator Analysis

PHPK Technologies Incorporated

(LLNL Technical Contact: Brian Felker)

February 10, 1995

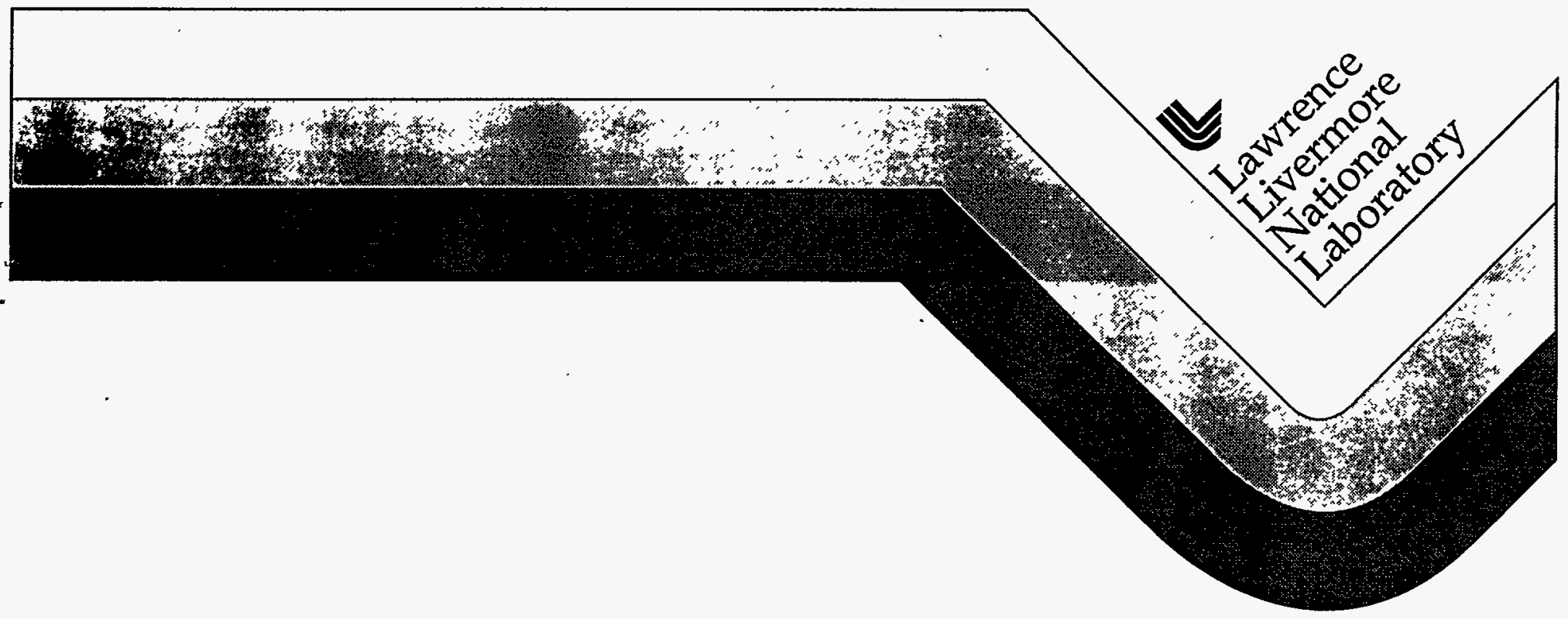




\section{DISCLAIMER}

Work performed under the auspices of the U.S. Department of Energy by Lawrence Livermore National Laboratory under contract number W-7405-ENG-48.

This document was prepared as an account of work sponsored by an agency of the United States Government. Neither the United States Government nor the University of California nor any of their employees, makes any warranty, express or implied, or assumes any legal liability or responsibility for the accuracy, completeness, or usefulness of any information, apparatus, product, or process disclosed, or represents that its use would not infringe privately owned rights. Reference herein to any specific commercial products, process, or service by trade name, trademark, manufacturer, or otherwise, does not necessarily constitute or imply its endorsement, recommendation, or favoring by the United States Government or the University of California. The views and opinions of authors expressed herein do not necessarily state or reflect those of the United States Government or the University of California, and shall not be used for advertising or product endorsement purposes. 


\section{DISCLAIMIER}

Portions of this document may be illegible in electronic image products. Images are produced from the best available original document. 


\title{
MFTF-B REFRIGERATOR ANALYSIS
}

\author{
LLNL SUBCONTRACT B304356
}

PHPK TECHNOLOGIES INC. 


\subsection{Introduction}

The purpose of this analysis was to determine the applicability of the MFTF-B helium refrigerator to the requirements of the TPX Tokomak at Princeton. The TPX requires a high pressure stream of supercritical gas to all loads rather than the liquid helium loads that the refrigerator was originally designed to support.

\subsection{Load Comparison}

This study is based on the MFTF-B state point table, which is table 2.6.1.3-l of the LLNL Systems Design Description for the conceptual design review of March 29, 1993. It is assumed to be the original design table for the refrigerator, but is not based on operating data.

This table indicates a design load of 8800 watts and a delivery of $33.32 \mathrm{~g} / \mathrm{s}$ of liquid helium at $4.35 \mathrm{~K}$.

In contrast to this, the TPX will require a stream of supercritical gas at temperatures between 4.2 and $5 \mathrm{~K}$ and at pressures between 4 and 10 atmospheres. $24 \mathrm{~g} / \mathrm{s}$ of this stream flows to the power leads and is returned to the system at room temperature. The remainder absorbs the magnet heat load and returns at temperatures between 6 and $9 \mathrm{~K}$. The magnet loads are as follows:

- Initial load - 5750 watts

- Second year - 7480 watts

- Final maximum load - 8350 watts

\subsection{Cycle Duplication}

The first step in this analysis was to duplicate as closely as possible the original state point table for the refrigerator. The duplication state point table is shown in the appendix, together with the schematic diagram. The high pressure compressor flow in the duplication is $1002 \mathrm{~g} / \mathrm{s}$, compared to $993 \mathrm{~g} / \mathrm{s}$ in the original table, an error of $0.9 \%$. Our present programs use a direct computer code to calculate helium properties based on the HEPAK program developed by Cryodata, which is in turn based on revised versions of NIST Technical Note 1334 (1991) Part of the discrepancy could therefore be due to our use of more recent data.

The duplication provides the design UA values for the heat exchangers and the flow coefficients for the expanders, which permit the calculation of off-design operation. These values are also shown in the appendix. 
The heat exchanger table shows total load in watts and btu, the log mean temperature difference in degrees $K$ and the UA value in watts $K$.

\subsection{Cycle Simulation}

\subsection{Simulation method}

The principle changes which occur when these cycles are operated off-design involve the heat exchangers and expanders. The pressure drops through the system change only slightly, and for purposes of this study have been ignored. The UA values for the heat exchangers are assumed to vary as the .8 power of the flow rate, using the flow values of the original cycle as a basis.

The expander calculation is somewhat more complex. For expanders \#1 and \#2 where expansion ratio is greater than four, it is assumed that the expander operates as a sonic orifice, and real gas orifice equations are used to calculate the flow, based on the input temperature and pressure, and the flow constants referred to above. For turbine \#3 the flow is subsonic and the downstream pressure affects the flow. An excellent correlation was obtained from Air Liquide for this computation which represents the best information available at present for these very low temperatures.

The efficiency of the expanders can also be expected to vary with flow rate, and prediction of this effect is very difficult. Some loss of efficiency can be expected as the flow varies in either direction from the design point. With no actual data available for this. the following relationship was included in the programs:

$\%$ actual eff $=\%$ design eff $-10 \times A B S(1-$ actual flow/design flow $)$

This gives a degradation in efficiency of about 3 percentage points for a $25 \%$ change in flow rate.

For this study, with no actual data on the compressors, they are not included in the simulation. For each case, however, the compressor loading is calculated based on intake volume only. This is calculated as a percent of maximum capacity, assuming that the compressors are designed to be fully loaded at the MFTF-B design point. 


\subsection{Modified MFTF-B Cycle}

The modified cycle schematic is shown in the appendix. Only the lower section below heat exchanger \#8 is changed. Using the techniques described above, a simulation program was written, using the heat exchangers and the two warm turbines of the original cycle. Since the principle change is at the cold end, it was assumed that the \#3 expander would have to be replaced, and its characteristics were chosen to match the new load requirements. The design point was for the maximum load that the system would support with 10 atmospheres load pressure and compressor flows as close as possible to the MFTF-B flows. This turned out to be a load of 7850 watts and $24 \mathrm{~g} / \mathrm{s}$ lead flow, with compressor flows of $98.9 \%$ and $101.4 \%$ of the first and second stage MFTF-B flows. We received performance specifications for the \#3 turbine from Air Liquide and from Lotepro, indicating efficiencies of $75 \%$ and $71 \%$ respectively. We chose to use $74 \%$ in the simulation. The simulation state point table is shown in the appendix.

\subsection{Modified Cycle Simulation}

Using the flow characteristics of turbine \#3, and the Air Liquide equations, a complete simulation program was written to enable the calculation of other operating points. With this program it is possible to change any of the cycle parameters, including loads, pressures, throttling of the turbines, etc., to predict as closely as possible the operating conditions. The program creates a complete state point table for each case. Since the available choices are very large, only selected cases were run for this study. It was also considered unciesirable to throttle any of the turbines due to the resulting efficiency loss, so all cases were run with the turbines at full flow.

\subsection{Selected Operating Points}

Seven off-design cases were run to determine the capacity of the machine and illustrate the effect of changes in load pressure, both at high loading and at the initial expected load of 5750 watts. The conductor load was $24 \mathrm{~g} / \mathrm{s}$ in all cases. Results are shown in the following table and in the accompanying graphs. Complete state point tables for all cases are in the Appendix. 


\section{OFF-DESIGN PERFORMANCE}

\begin{tabular}{|c|c|c|c|c|c|}
\hline Case & Load & Load Press & Cycle Press & 1st Comp\% & 2nd Comp \% \\
\hline Design & 7850 & 10 & 19.85 & 98.90 & 101.42 \\
\hline$\# 1$ & 8850 & 8 & 20 & 107.66 & 105.75 \\
\hline \#2 & 9650 & 6 & 20 & 114.57 & 108.85 \\
\hline$\# 3$ & 10500 & 4 & 20 & 121.74 & 112.24 \\
\hline$\# 4$ & 5750 & 10 & 17.82 & 80.65 & 86.67 \\
\hline$\# 5$ & 6450 & 8 & 17.82 & 89.22 & 90.45 \\
\hline$\# 6$ & 7240 & 6 & 17.82 & 97.31 & 94.11 \\
\hline$\# 7$ & 8025 & 4 & 17.82 & 105.25 & 97.88 \\
\hline
\end{tabular}

High Load Series

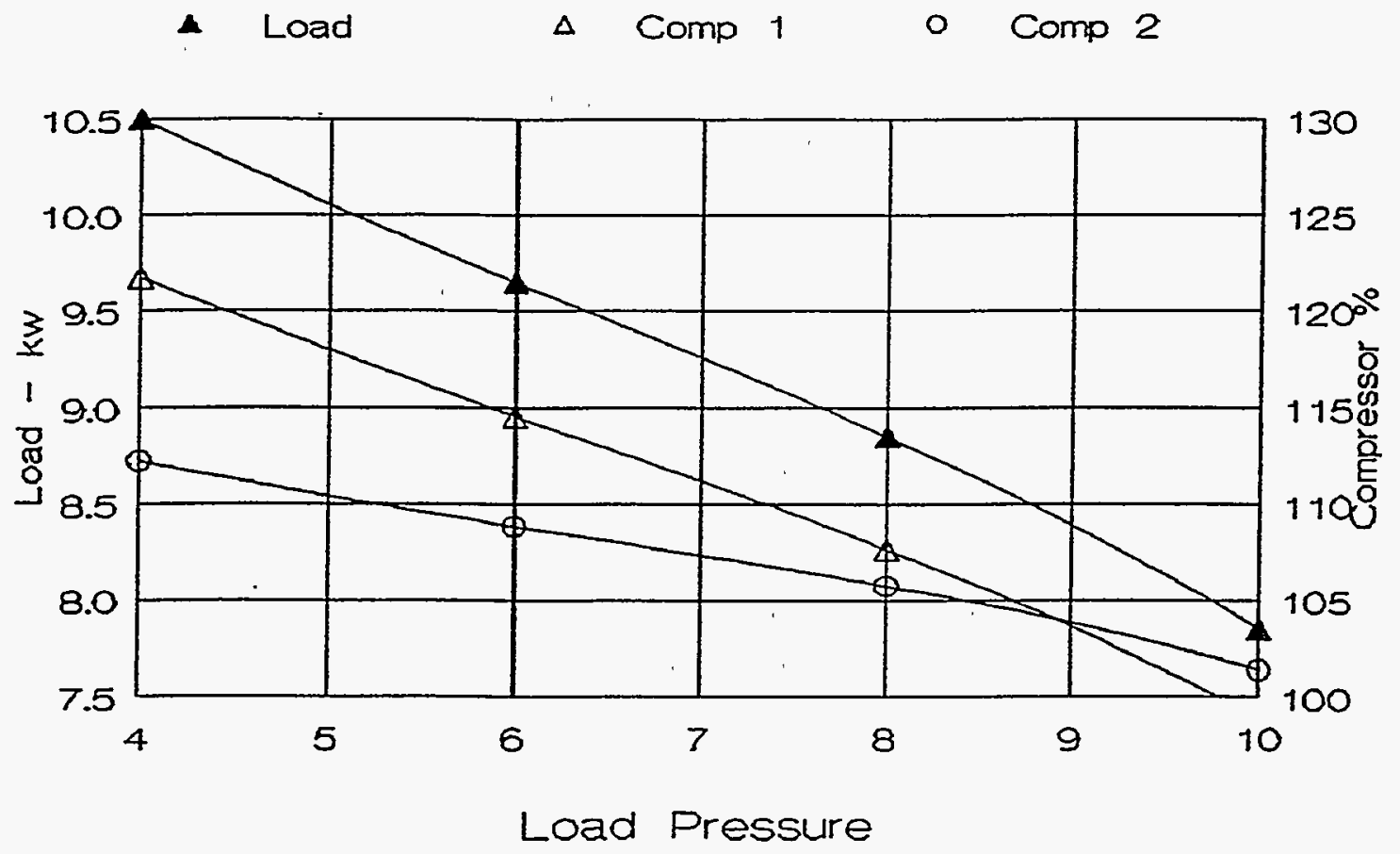

Page 4 


\section{Low Load Series}
- Load
$\triangle$ Comp 1
- Comp 2

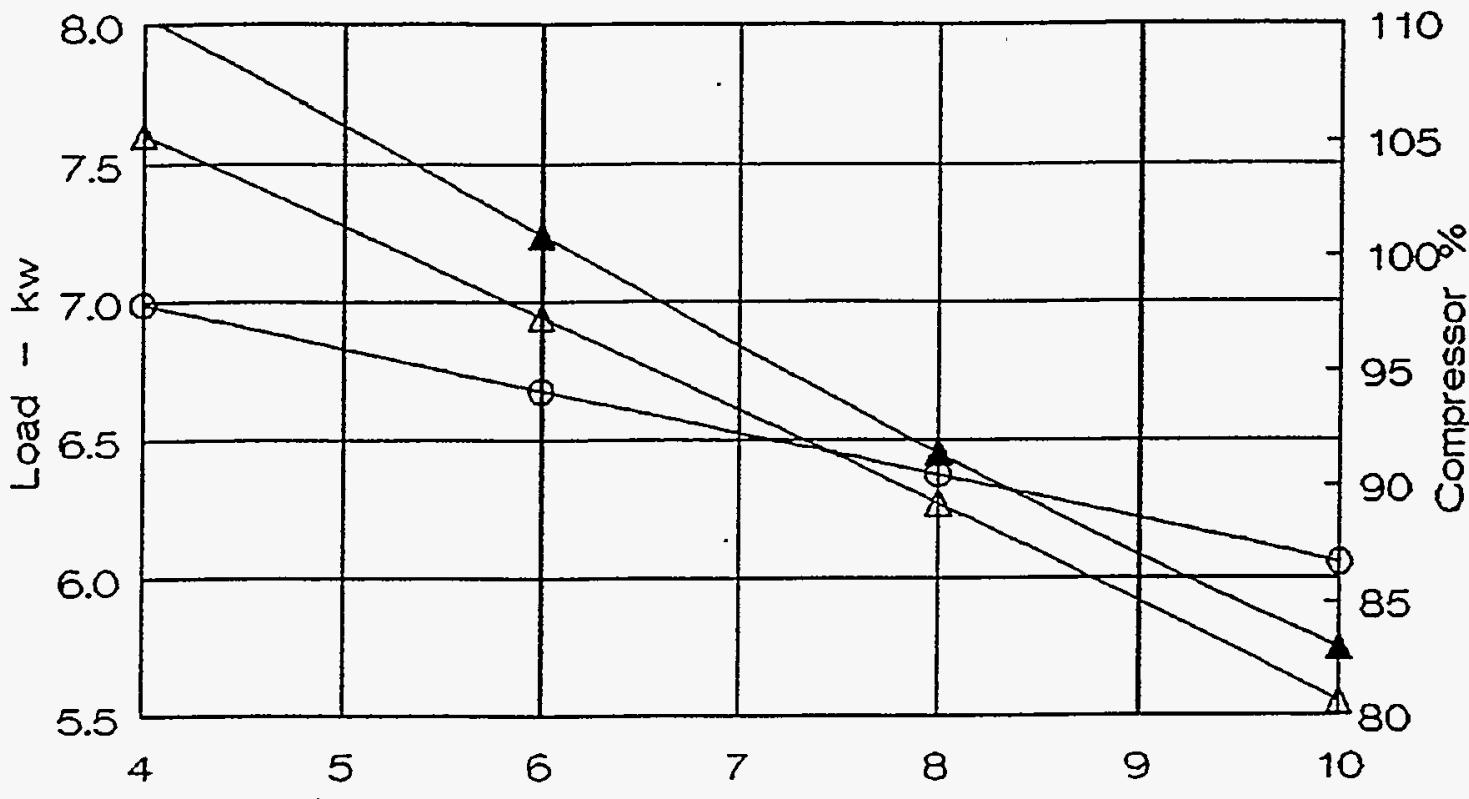

Load Pressure

\subsection{Conclusion}

The results indicate that with a properly chosen turbine the system will operate well under the desired conditions. Additional compressor capacity may be necessary for the higher loads expected in future years, but a single turbine should cover the load changes. This study is, of course, based on the original state point table, which was a design point, and not a measured operating point. To be certain of the results, actual operating data will be necessary to confirm the condition of the present system. 
MFTF-B SCHEMATIC

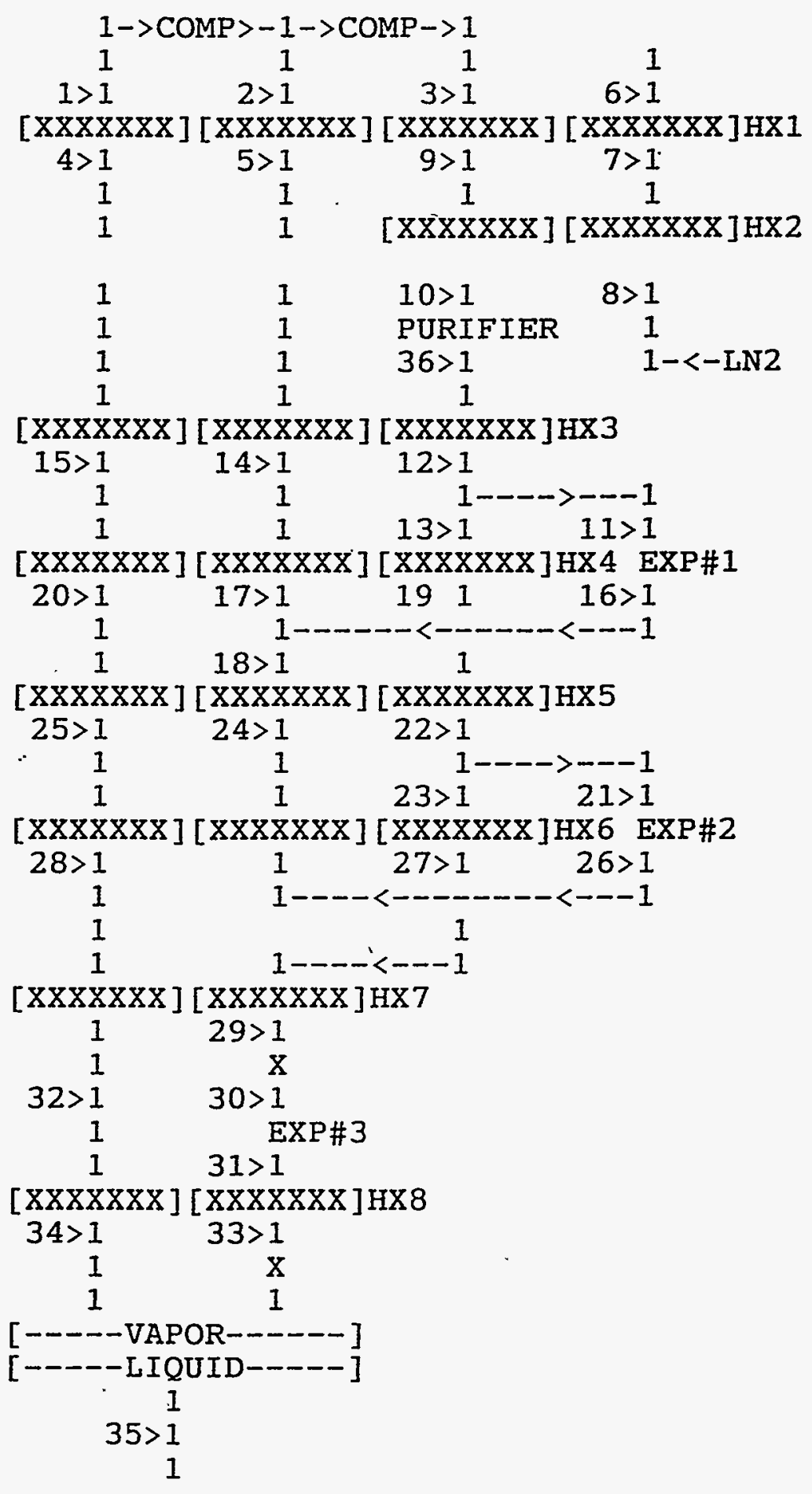


STATE POINT TABLE FOR MFTF-B CYCLE

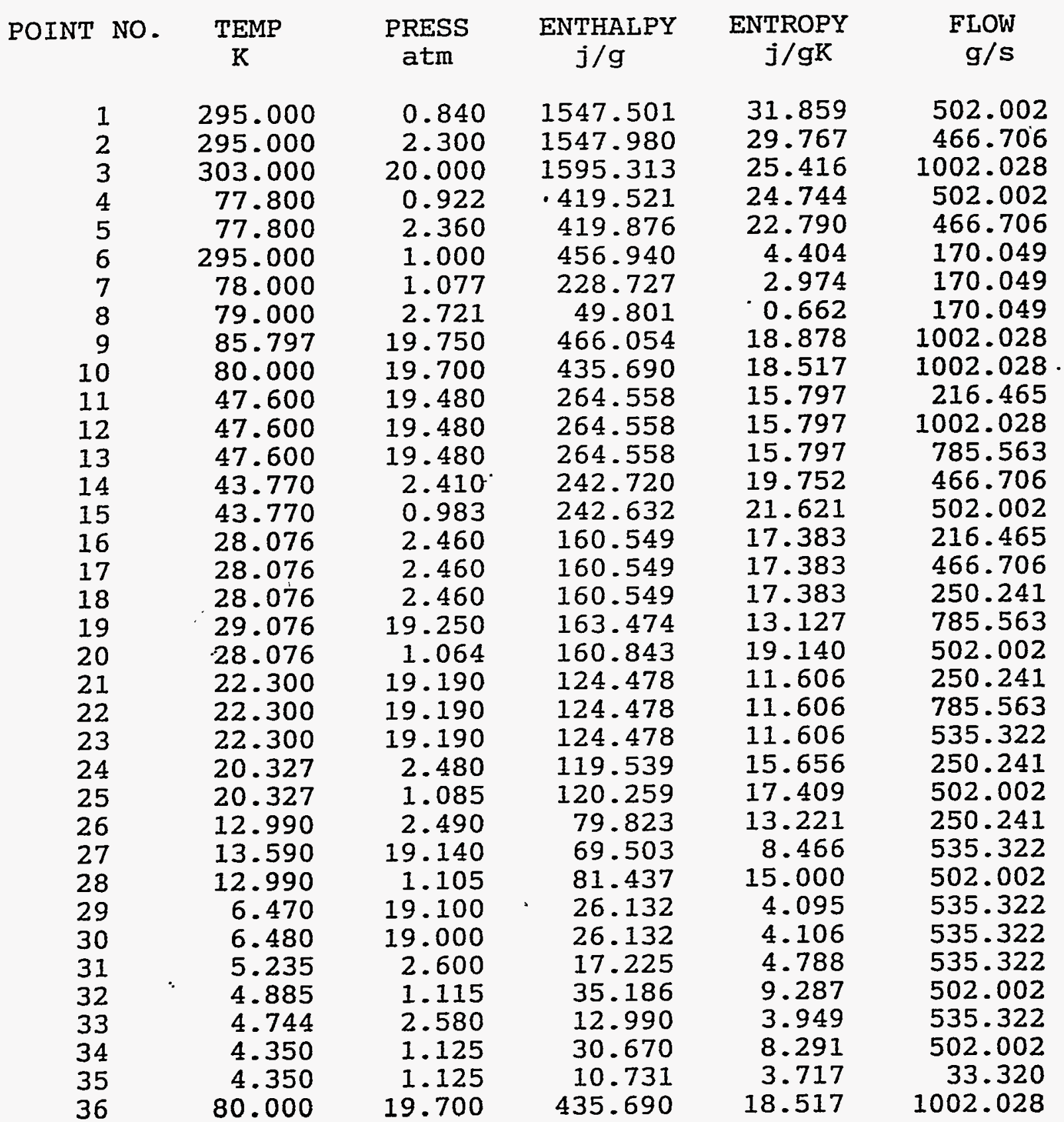

? 
HEAT EXCHANGER DATA FOR MFTF-B CYCLE

\begin{tabular}{rrrrr} 
HX\# & \multicolumn{1}{c}{ WATTS } & \multicolumn{1}{c}{ BTU } & LMTD & UA- $/ \mathrm{K}$ \\
1 & 1131549 & 3861977 & 8.00 & 141466.5 \\
2 & 30426 & 103844 & 3.31 & 9193.0 \\
3 & 171478 & 585256 & 2.94 & 58323.0 \\
4 & 79408 & 271018 & 2.11 & 37678.7 \\
5 & 30634 & 104555 & 1.43 & 21392.6 \\
6 & 29429 & 100441 & 1.15 & 25511.2 \\
7 & 23218 & 79243 & 1.01 & 22896.1 \\
8 & 2267 & 7739 & 0.37 & 6099.3
\end{tabular}

EXPANDER DATA FOR MFTF-B CYCLE

$\begin{array}{lrrrrr}\text { EXPANDER NO. } 1 & 22.51 \mathrm{KW} & \mathrm{K}=487.27 & \text { EFF'Y }^{\prime}=73.0 \% \\ \text { EXPANDER NO. } 2 & 11.17 \mathrm{KW} & \mathrm{K}=376.93 & \text { EFF'Y }^{\prime}=71.0 \% \\ \text { EXPANDER NO. } 3 & 4.77 \mathrm{KW} & \mathrm{K}=446.47 & \text { EFF'Y }^{\prime}=72.0 \%\end{array}$


SCHEMATIC FOR MFTF-B MODIFIED FOR THE TPX

$\because$<smiles>[3H]C=[V][3H]</smiles>

1

$1<42 \quad 1->$ COMP $>-1->$ COMP $\rightarrow 1$

$1 \quad 1>1 \quad 2>1 \quad 3>1 \quad 6>1$.

$1[\mathrm{XXXXXXX}][\mathrm{XXXXXXX}][\mathrm{XXXXXXX}][\mathrm{XXXXXXX}] \mathrm{HX}]$ $4>1$

1

$1 \quad 1 \quad[\mathrm{XXXXXXX}][\mathrm{XXXXXXX}] \mathrm{HX} 2$

$5>1$

$9>1$

$7>1$

$1 \quad 1 \quad 10>1 \quad 8>1$

$1 \quad 1 \quad$ PURIFIER 1

$1 \quad 1 \quad 36>1 \quad 1-<-$ IN2

$1 \quad 1 \quad i$

[XXXXXX] ] $\mathrm{XXXXXXX}][\mathrm{XXXXXXX}] \mathrm{HX} 3$

$15>1 \quad 14>1 \quad 12>1$

$\begin{array}{lccc}1 & 1 & 1 & 1--->--1 \\ 1 & 1 & 13>1 & 11>1\end{array}$

[XXXXXXX] $[\mathrm{XXXXXXX}][\mathrm{XXXXXXX}] \mathrm{HX} 4$ EXP\#1

$\begin{array}{lllll}20>1 & 17>1 & 19 & 1 & 16>1\end{array}$

$44>1$

1

$1-\cdots---<--\infty<--1$

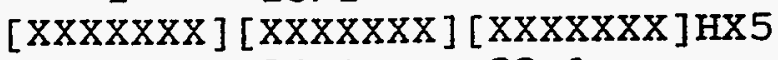

$\begin{array}{cccc}25>1 & 24>1 & 22>1 & \\ 1 & 1 & 1 & 1-0>-1 \\ 1 & 1 & 23>1 & 21>1 \\ {[\mathrm{XXXXXXX}][\mathrm{XXXXXXX}][\mathrm{XXXXXX}] \mathrm{HX6}} & \text { EXP\#2 }\end{array}$

$28>1$

$43>1$

$27>1$

$26>1$

1

$45>1----<------<--1$

$1----<---1$

$[\mathrm{XXXXXXX}][\mathrm{XXXXXXX}] \mathrm{HX} 7$

$1 \quad 29>1$

$\begin{array}{rr}1 & x \\ 1 & 30>1\end{array}$

$1 \quad$ EXP\#3

$1 \quad 31>1$

[ $\mathrm{XXXXXXX}][\mathrm{XXXXXXX}] \mathrm{HX} 8$

$:$

$\stackrel{0}{9}$

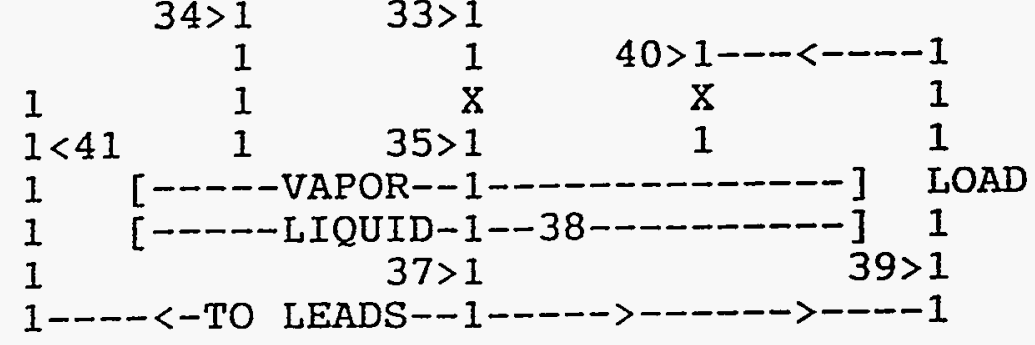


DESIGN POINT TABLE - MFTF-B MODIFIED FOR THE TPX

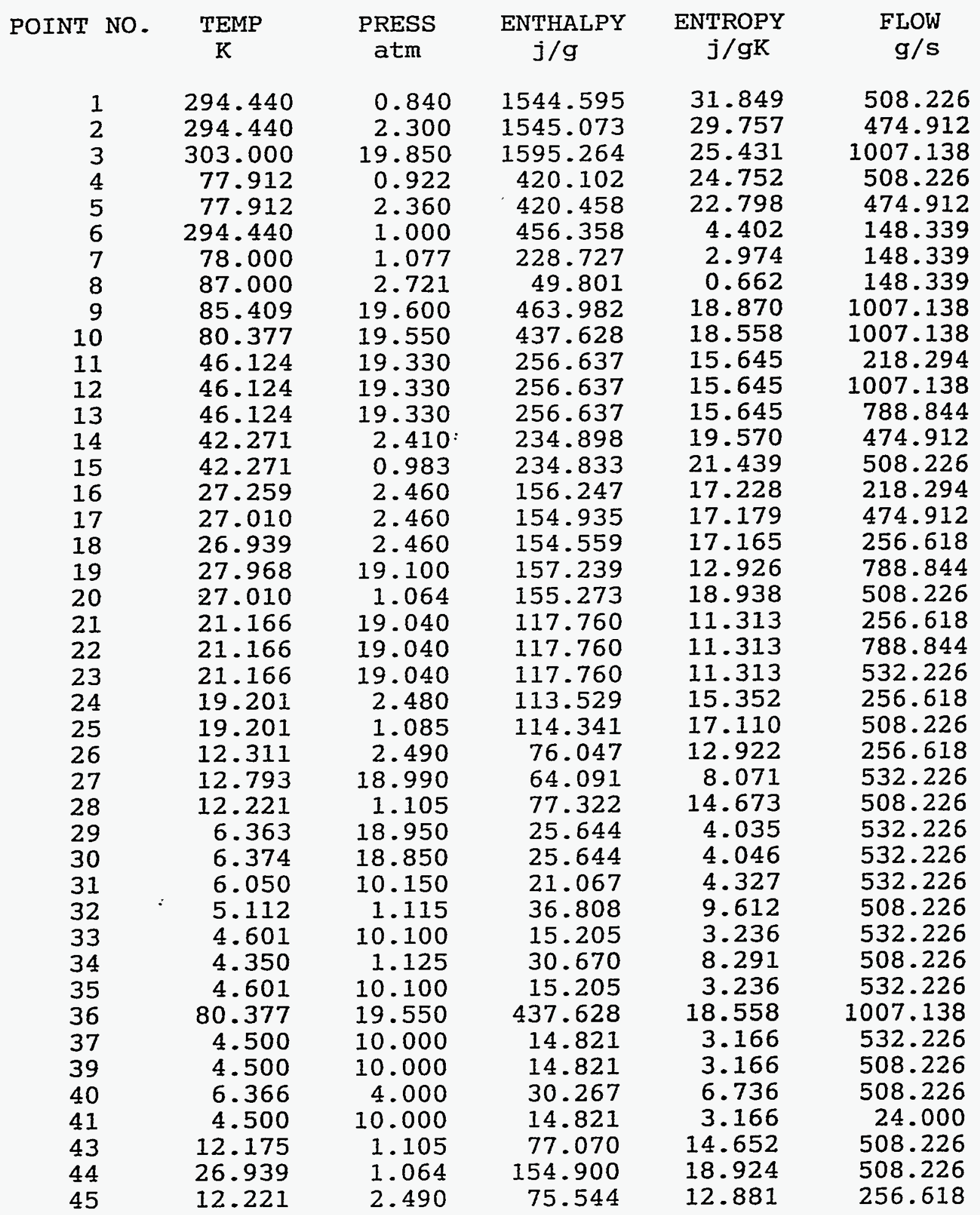


OFF-DESIGN CASE \#1

CYCLE PRESSURE 20 ATM LOAD PRESSURE 8 ATM $24 \mathrm{G} / \mathrm{S} \quad 8850$ WATTS

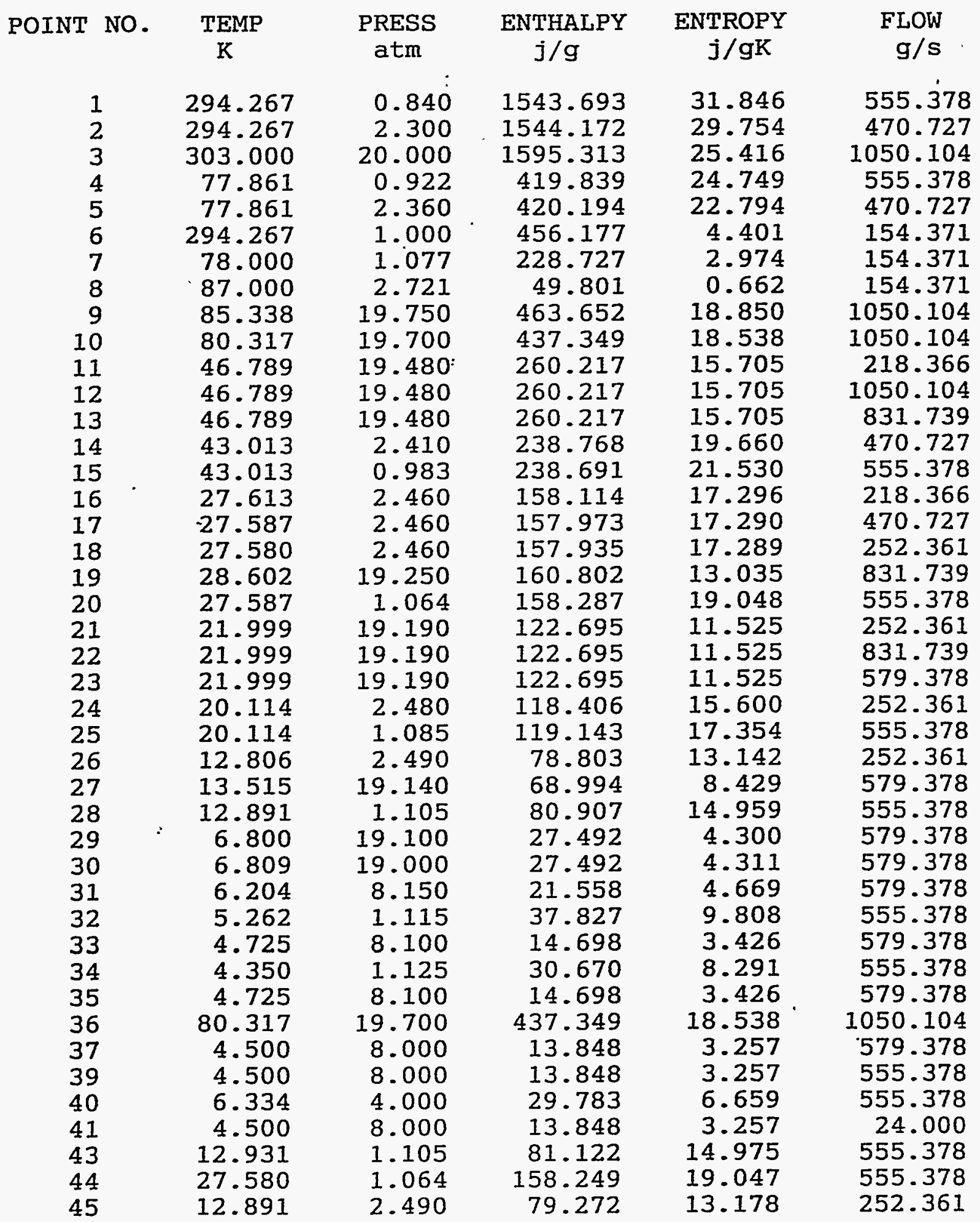


OFF-DESIGN CASE \#2

CYCLE PRESSURE 20 ATM LOAD PRESSURE 6 ATM $24 \mathrm{G} / \mathrm{S} 9650$ WATTS

\begin{tabular}{|c|c|c|c|c|c|}
\hline POINT NO. & $\underset{K}{\text { TEMP }}$ & $\begin{array}{l}\text { PRESS } \\
\text { atm }\end{array}$ & $\begin{array}{c}\text { ENTHALPY } \\
j / g\end{array}$ & $\begin{array}{c}\text { ENTROPY } \\
j / g K\end{array}$ & $\begin{array}{r}\text { FLOW } \\
\text { g/s }\end{array}$ \\
\hline 1 & 294.154 & 0.840 & 1543.106 & 31.844 & 592.578 \\
\hline 2 & 294.154 & 2.300 & 1543.584 & 29.752 & 464.322 \\
\hline 3 & 303.000 & 20.000 & 1595.313 & 25.416 & 1080.899 \\
\hline 4 & 78.060 & 0.922 & 420.870 & 24.762 & 592.578 \\
\hline 5 & 78.060 & 2.360 & 421.226 & 22.808 & 464.322 \\
\hline 6 & 294.154 & 1.000 & 456.060 & 4.401 & 158.535 \\
\hline 7 & 78.000 & 1.077 & 228.727 & 2.974 & 158.535 \\
\hline 8 & 87.000 & 2.721 & 49.801 & 0.662 & 158.535 \\
\hline 9 & 85.519 & 19.750 & 464.599 & 18.861 & 1080.899 \\
\hline 10 & 80.509 & 19.700 & 438.356 & 18.550 & 1080.899 \\
\hline 11 & 47.458 & $19.480^{\circ}$ & 263.797 & 15.781 & 216.804 \\
\hline 12 & 47.458 & 19.480 & 263.797 & 15.781 & 1080.899 \\
\hline 13 & 47.458 & 19.480 & 263.797 & 15.781 & 864.095 \\
\hline 14 & 43.738 & 2.410 & 242.553 & 19.748 & 464.322 \\
\hline 15 & 43.738 & 0.983 & 242.465 & 21.617 & 592.578 \\
\hline 16 & 27.995 & 2.460 & 160.120 & 17.368 & 216.804 \\
\hline 17 & 28.122 & 2.460 & 160.791 & 17.392 & 464.322 \\
\hline 18 & 28.155 & 2.460 & 160.965 & 17.398 & 247.517 \\
\hline 19 & 29.179 & 19.250 & 164.052 & 13.147 & 864.095 \\
\hline 20 & 28.122 & 1.064 & 161.083 & 19.149 & 592.578 \\
\hline 21 & 22.693 & 19.190 & 126.802 & 11.709 & 247.517 \\
\hline 22 & $22.693^{\circ}$ & 19.190 & 126.802 & 11.709 & 864.095 \\
\hline 23 & 22.693 & 19.190 & 126.802 & 11.709 & 616.578 \\
\hline 24 & 20.859 & 2.480 & 122.377 & 15.794 & 247.517 \\
\hline 25 & 20.859 & 1.085 & 123.057 & 17.545 & 592.578 \\
\hline 26 & 13.255 & 2.490 & 81.290 & 13.333 & 247.517 \\
\hline 27 & 14.122 & 19.140 & 73.113 & 8.727 & 616.578 \\
\hline 28 & 13.452 & 1.105 & 83.904 & 15.186 & 592.578 \\
\hline 29 & 7.073 & 19.100 & 28.681 & 4.472 & 616.578 \\
\hline 30 & 7.081 & 19.000 & 28.681 & 4.482 & 616.578 \\
\hline 31 & 6.134 & 6.150 & 21.496 & 4.942 & 616.578 \\
\hline 32 & 5.306 & 1.115 & 38.128 & 9.865 & 592.578 \\
\hline 33 & 4.844 & 6.100 & 14.328 & 3.653 & 616.578 \\
\hline 34 & 4.350 & 1.125 & 30.670 & 8.291 & 592.578 \\
\hline 35 & 4.844 & 6.100 & 14.328 & 3.653 & 616.578 \\
\hline 36 & 80.509 & 19.700 & 438.356 & 18.550 & 1080.899 \\
\hline 37 & 4.500 & 6.000 & 12.927 & 3.369 & 616.578 \\
\hline 39 & 4.500 & 6.000 & 12.927 & 3.369 & 592.578 \\
\hline 40 & 6.297 & 4.000 & 29.211 & 6.569 & 592.578 \\
\hline 41 & 4.500 & 6.000 & 12.927 & 3.369 & 24.000 \\
\hline 43 & 13.537 & 1.105 & 84.360 & 15.220 & 592.578 \\
\hline 44 & 28.155 & 1.064 & 161.257 & 19.155 & 592.578 \\
\hline 45 & 13.452 & 2.490 & 82.378 & 13.414 & 247.517 \\
\hline
\end{tabular}


OFF-DESIGN CASE \#3

CYCLE PRESSURE 20 ATM LOAD PRESSURE 4 ATM $24 \mathrm{G} / \mathrm{S} \quad 10500$ WATTS

\begin{tabular}{|c|c|c|c|c|c|}
\hline POINT NO. & $\begin{array}{l}\text { TEMP } \\
\mathrm{K}\end{array}$ & $\begin{array}{l}\text { PRESS } \\
\text { atm }\end{array}$ & $\begin{array}{c}\text { ENTHALPY } \\
j / g\end{array}$ & $\begin{array}{c}\text { ENTROPY } \\
\mathrm{j} / \mathrm{gK}\end{array}$ & $\begin{array}{l}\text { FLOW } \\
\text { g/s }\end{array}$ \\
\hline 1 & 294.016 & 0.840 & 1542.391 & 31.842 & 631.170 \\
\hline 2 & 294.016 & 2.300 & 1542.869 & 29.750 & 459.385 \\
\hline 3 & 303.000 & 20.000 & 1595.313 & 25.416 & 1114.555 \\
\hline 4 & 77.907 & 0.922 & 420.077 & 24.752 & 631.170 \\
\hline 5 & 77.907 & 2.360 & 420.433 & 22.797 & 459.385 \\
\hline 6 & 294.016 & 1.000 & 455.916 & 4.400 & 163.715 \\
\hline 7 & 78.000 & 1.077 & 228.727 & 2.974 & 163.715 \\
\hline 8 & 87.000 & 2.721 & 49.801 & 0.662 & 163.715 \\
\hline 9 & 85.356 & 19.750 & 463.744 & 18.851 & 1114.555 \\
\hline 10 & 80.338 & 19.700 & 437.462 & 18.539 & 1114.555 \\
\hline 11 & 47.933 & $19.480^{\circ}$ & 266.338 & 15.834 & 215.717 \\
\hline 12 & 47.933 & 19.480 & 266.338 & 15.834 & 1114.555 \\
\hline 13 & 47.933 & 19.480 & 266.338 & 15.834 & 898.838 \\
\hline 14 & 44.282 & 2.410 & 245.393 & 19.812 & 459.385 \\
\hline 15 & 44.282 & 0.983 & 245.297 & 21.681 & 631.170 \\
\hline 16 & 28.285 & 2.460 & 161.650 & 17.422 & 215.717 \\
\hline 17 & -28.576 & 2.460 & 163.181 & 17.476 & 459.385 \\
\hline 18 & 28.649 & 2.460 & 163.561 & 17.489 & 243.669 \\
\hline 19 & 29.677 & 19.250 & 166.852 & 13.242 & 898.838 \\
\hline 20 & 28.576 & 1.064 & 163.456 & 19.233 & 631.170 \\
\hline 21 & 23.278 & 19.190 & 130.243 & 11.859 & 243.669 \\
\hline 22 & 23.278 & 19.190 & 130.243 & 11.859 & 898.838 \\
\hline 23 & 23.278 & 19.190 & 130.243 & 11.859 & 655.170 \\
\hline 24 & 21.481 & 2.480 & 125.686 & 15.950 & 243.669 \\
\hline 25 & 21.481 & 1.085 & 126.322 & 17.699 & 631.170 \\
\hline 26 & 13.649 & 2.490 & 83.463 & 13.494 & 243.669 \\
\hline 27 & 14.576 & 19.140 & 76.167 & 8.940 & 655.170 \\
\hline 28 & 13.855 & 1.105 & 86.054 & 15.344 & 631.170 \\
\hline 29 & 7.061 & 19.100 & 28.628 & 4.464 & 655.170 \\
\hline 30 & 7.069 & 19.000 & 28.628 & 4.474 & 655.170 \\
\hline 31 & 5.765 & 4.150 & 20.214 & 5.049 & 655.170 \\
\hline 32 & 5.163 & 1.115 & 37.158 & 9.680 & 631.170 \\
\hline 33 & 4.896 & 4.100 & 13.963 & 3.894 & 655.170 \\
\hline 34 & 4.350 & 1.125 & 30.670 & 8.291 & 631.170 \\
\hline 35 & 4.896 & 4.100 & 13.963 & 3.894 & 655.170 \\
\hline 36 & 80.338 & 19.700 & 437.462 & 18.539 & 1114.555 \\
\hline 37 & 4.500 & 4.000 & 12.113 & 3.517 & 655.170 \\
\hline 39 & 4.500 & 4.000 & 12.113 & 3.517 & 631.170 \\
\hline 40 & 6.268 & 4.000 & 28.749 & 6.495 & 631.170 \\
\hline 41 & 4.500 & 4.000 & 12.113 & 3.517 & 24.000 \\
\hline 43 & 13.940 & 1.105 & 86.505 & 15.376 & 631.170 \\
\hline 44 & 28.649 & 1.064 & 163.834 & 19.246 & 631.170 \\
\hline 45 & 13.855 & 2.490 & 84.599 & 13.577 & 243.669 \\
\hline
\end{tabular}


OFF-DESIGN CASE \#4

CYCLE PRESSURE 17.82 ATM $24 \mathrm{G} / \mathrm{S} 5750$ WATTS

POINT NO. TEMP

K

PRESS

atm

0.840

2.300

17.820

0.922

2.360

1.000

1.077

2. 721

17.570

17.520

17.300

17.300

17.300

2. 410

0.983

2. 460

2.460

2. 460

17.070

1.064

17.010

17.010

17.010

2. 480

1.085

2.490

16.960

1.105

16.920

16.820

10.150

1.115

10.100

1.125

10.100

17.520

10.000

10.000

4.000

10.000

1. 105

I. 064

2. 490
LOAD PRESSURE 10 ATM.

$\begin{array}{ccr}\text { ENTHALPY } & \text { ENTROPY } & \text { FLOW } \\ j / g & j / g K & g / s\end{array}$

$\begin{array}{rr}1547.626 & 31.860 \\ 1548.105 & 29.768 \\ 1594.599 & 25.655 \\ 420.630 & 24.759 \\ 420.987 & 22.804 \\ 456.965 & 4.404 \\ 228.727 & 2.974 \\ 49.801 & 0.662 \\ 464.267 & 19.108\end{array}$

464.267

437.062

254.281

254.281

254.281

232.816

232.758

159.558

156.186

155.145

157.965

156.513

117.785

117.785

117.785

113.150

113.969

77.988

68.404

80.777

31.195

31.195

27.284

42.037

16.545

30.670

16.545

437.062

14.821

14.821

28.845

14.821

81.424

155.481

79.138
18.786

15.835

15.835

15.835

19.520

21.390

17.348

17.225

17.187

13.196

18.984

11.554

11.554

11.554

15.332

17.090

13.078

8.618

14.949

5.032

5.043

5.268

10.563

3.515

8.291

3.515

18.786

3.166

3.166

6.510

3.166

14.999

18.946

13.168
410.035

426.603

860.638

410.035

426.603

130.860

130.860 .

130.860

860.638

860.638

196.638

860.638

664.001

426.603

410.035

196.638

426.603

229.966

664.001

410.035

229.966

664.001

434.035

229.966

410.035

229.966

434.035

410.035

434.035

434.035

434.035

410.035

434.035

410.035

434.035

860.638

434.035

410.035

410.035

24.000

410.035

410.035

229.966 
OFF-DESIGN CASE \#5

CYCLE. PRESSURE 17.82 ATM 24 G/S 6450 WATTS

$\begin{array}{cccr}\text { PRESS } & \text { ENTHALPY } & \text { ENTROPY } & \text { FLOW } \\ \text { atm } & j / g & j / g K & g / s\end{array}$

POINT NO. TEMP

$\begin{array}{rr}1 & 294.844 \\ 2 & 294.844 \\ 3 & 303.000 \\ 4 & 78.031 \\ 5 & 78.031 \\ 6 & 294.844 \\ 7 & 78.000 \\ 8 & 87.000 \\ 9 & 85.545 \\ 10 & 80.372 \\ 11 & 46.605 \\ 12 & 46.605 \\ 13 & 46.605 \\ 14 & 42.856 \\ 15 & 42.856 \\ 16 & 28.456 \\ 17 & 28.003 \\ 18 & 27.872 \\ 19 & 28.875 \\ 20 & 28.003 \\ 21 & 22.098 \\ 22 & 22.098 \\ 23 & 22.098 \\ 24 & 20.244 \\ 25 & 20.244 \\ 26 & 13.367 \\ 27 & 14.142 \\ 28 & 13.666 \\ 29 & 8.036 \\ 30 & 8.039 \\ 31 & 7.040 \\ 32 & 5.952 \\ 33 & 5.160 \\ 34 & 4.350 \\ 35 & 5.160 \\ 36 & 80.372 \\ 37 & 4.500 \\ 39 & 4.500 \\ 40 & 6.221 \\ 41 & 4.500 \\ 43 & 13.817 \\ 44 & 27.872 \\ 45 & 13.666\end{array}$

LOAD PRESSURE 8 ATM

$$
0.840
$$

2.300

17.820

0.922

2. 360

1.000

1.077

2.721

17.570

17.520

$17.300^{\circ}$

17.300

17.300

2.410

0.983

2.460

2.460

2.460

17.070

1.064

17.010

17.010

17.010

2.480

1.085

2. 490

16.960

1. 105

16.920

16.820

8.150

1.115

8.100

1. 125

8.100

17.520

8.000

8.000

4.000

8.000

1. 105

1.064

2.490
31.856

29.764

25.655

24.760

22.806

4.403

2.974

0.662

19.107

18.786

15.937 .

15.937

15.937

19.641

21.511

17.454

17.369

17.345

13.357

19.127

11.835

11.835

11.835

15.635

17.388

13.379

9.046

15.270

5. 244

5.254

5.551

10.601

3.770

8.291

3.770

18.786

3.257

3.257

6.373

3.257

15.329

19.102

13.501
1546.690

1547.168

1594.599

420.723

421.079

456.777

228.727

49.801

464.159

437.086

258.993

258.993

258.993

237.950

237.875

162.547

160.162

159.476

162.563

160.459

123.840

123.840

123.840

119.100

119.827

81.911

74.273

85.044

32.865

32.865

27.412

42.263

16.397

30.670

16.397

437.086

13.848

13.848

27.986

13.848

85.850

159.778

83.556
456.213

417.916

898.129

456.213

417.916

135.895

135.895 .

135.895

898.129

898.129

194.744

898.129

703.386

417.916

456.213

194.744

417.916

223.172

703.386

456.213

223.172

703.386

480.213

223.172

456.213

223.172

480.213

456.213

480.213

480.213

480.213

456.213

480.213

456.213

480.213

898.129

480.213

456.213

456.213

24.000

456.213

456.213

223.172 
OFF-DESIGN CASE \#6

CYCLE PRESSURE 17.82 ATM $24 \mathrm{G} / \mathrm{S} \quad 7240$ WATTS
LOAD PRESSURE 6 ATM

$\begin{array}{ccc}\text { PRESS } & \text { ENTHALPY : } & \text { ENTROPY } \\ \text { atm } & j / g & j / g K\end{array}$

FLOW

$\mathrm{g} / \mathrm{s}$

POINT NO. TEMP

1
2
3
4
5

1
2
3
4
5
6
7

8

9

10

11

12

13

14

15

16

17

18

19

20

21

22

23

24

25

26

27

28

29

30

31

32

33

34

35

36

37

39

40

41

43

44

45
294.686

294.686

303.000

78.139

78.139

294.686

78.000

87.000

85.617

80.470

47.425

47.425

47.425

43.760

43.760

28.984

28.698

28.621

29.631

28.698

22.984

22.984

22.984

21.187

21.187

13.977

14.846

14.310

8.070

8.072

6.637

5.774

5.225

4.350

5.225

80.470

4.500

4.500

6.187

4.500

14.462

28.621

14.310
$0.840 \quad 1545.872$

$2.300 \quad 1546.350$

$17.820 \quad 1594.599$

0.922

2.360

1.000

1.077

2.721

17.570

17.520

$17.300^{\circ}$

17.300

17.300

2.410

0.983

2.460

2.460

2.460

17.070

1.064

17.010

17.010

17.010

2.480

1.085

16.960

1.105

16.920

16.820

6.150

1. 115

6.100

1.125

6.100

17.520

6.000

6.000

4.000

6.000

1. 105

1.064

2. 490
421.283

421.639

456.614

228.727

49.801

464.532

437.597

263.368

263.368

263.368

242.671

242.582

165.323

163.823

163.418

166.788

164.094

129.021

129.021

129.021

124.121

124.778

85.271

78.978

88.475

33.053

33.053

26.032

41.151

16.031

30.670

16.031

437.597

12.927

12.927

27.416

12.927

89.282

163.691

87.096
2. 490
31.854

29.762

25.655

2.4 .767

22.813

4.402

2. 974

0.662

19.111

18.793

16.030

16.030

19.750

21.620

17.550

17.498

17.484

13.502

19.255

12.065

12.065

12.065

15.877

17.627

13.625

9.371

15.516

5.268

5.278

5.652

10.412

3.991

8. 291

3.991

18.793

3.369

3.369

6.281

3.369

15.572

19.241

13.754
16.030
499.672

410.854

934.526

499.672

410.854

140.682

140.682 .

140.682

934.526

934.526

193.034

934.526

741.492

410.854

499.672

193.034

410.854

217.820

741.492

499.672

217.820

741.492

523.672

217.820

499.672

217.820

523.672

499.672

523.672

523.672

523.672

499.672

523.672

499.672

523.672

934.526

523.672

499.672

499.672

24.000

499.672

499.672

217.820 
OFF-DESIGN CASE \#7

CYCLE PRESSURE 17.82 ATM LOAD PRESSURE 4 ATM $24 \mathrm{G} / \mathrm{S} \quad 8025$ WATTS

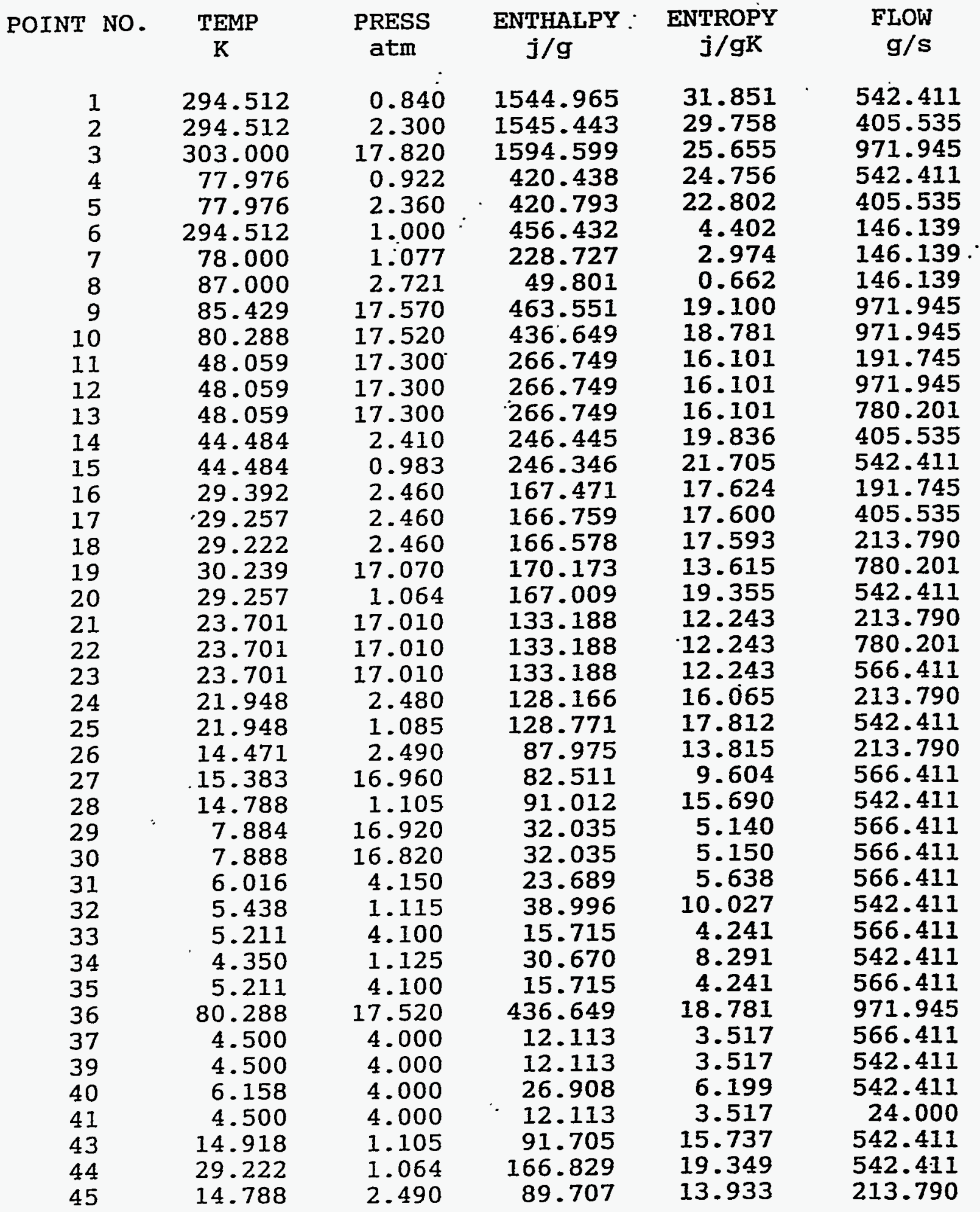


Technical Information Department - Lawrence Livermore National Laboratory University of California - Livermore, California 94551

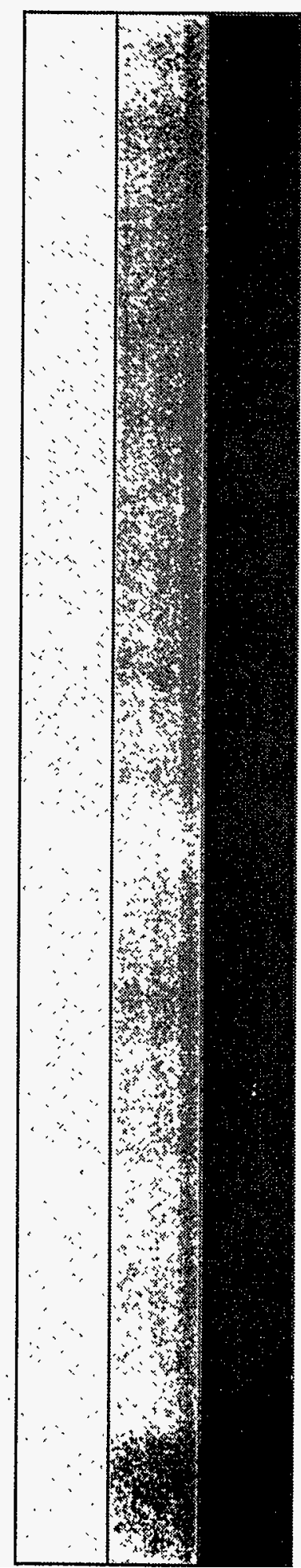

